\title{
Anterior Cingulate Cortico-Hippocampal Dysconnectivity in Unaffected Relatives of Schizophrenia Patients: A Stochastic Dynamic Causal Modeling Study
}

\author{
Yi-Bin $\mathrm{Xi}^{1 \dagger}$, Chen $\mathrm{Li}^{1 \dagger}$, Long-Biao Cui ${ }^{1+}$, Jian Liu ${ }^{2}$, Fan Guo ${ }^{1}$, Liang Li ${ }^{3}$, Ting-Ting Liu ${ }^{1}$, \\ Kang Liu ${ }^{1}$, Gang Chen ${ }^{1}$, Min $X^{4}{ }^{4}$, Hua-Ning Wang ${ }^{4}$ and Hong Yin ${ }^{1 *}$ \\ ${ }^{1}$ Department of Radiology, Xijing Hospital, Fourth Military Medical University, Xi'an, Shaanxi, China, ${ }^{2}$ Network Center, Fourth \\ Military Medical University, Xi'an, Shaanxi, China, ${ }^{3}$ School of Biomedical Engineering, Fourth Military Medical University, \\ Xi'an, Shaanxi, China, ${ }^{4}$ Department of Psychiatry, Xijing Hospital, Fourth Military Medical University, Xi'an, Shaanxi, China
}

OPEN ACCESS

Edited by:

Adeel Razi,

Wellcome Trust Centre for Neuroimaging (WT), UK

Reviewed by:

Sahil Bajaj,

The Houston Methodist Research Institute, USA

Wei Qin,

Xidian University, China

${ }^{*}$ Correspondence: Hong Yin

yinhong@fmmu.edu.cn

tThese authors have contributed equally to this work.

Received: 05 May 2016 Accepted: 14 July 2016

Published: 27 July 2016

Citation:

Xi Y-B, Li C, Cui L-B, Liu J, Guo F,

Li L, LiU T-T, LiU K, Chen G, Xi M, Wang H-N and Yin H (2016) Anterior

Cingulate Cortico-Hippocampal

Dysconnectivity in Unaffected Relatives of Schizophrenia Patients:

A Stochastic Dynamic Causal

Modeling Study.

Front. Hum. Neurosci. 10:383. doi: 10.3389/fnhum.2016.00383
Familial risk plays a significant role in the etiology of schizophrenia (SZ). Many studies using neuroimaging have demonstrated structural and functional alterations in relatives of SZ patients, with significant results found in diverse brain regions involving the anterior cingulate cortex (ACC), caudate, dorsolateral prefrontal cortex (DLPFC), and hippocampus. This study investigated whether unaffected relatives of first episode SZ differ from healthy controls (HCs) in effective connectivity measures among these regions. Forty-six unaffected first-degree relatives of first episode SZ patients-according to the DSM-IV - were studied. Fifty HCs were included for comparison. All subjects underwent resting state functional magnetic resonance imaging (fMRI). We used stochastic dynamic causal modeling (SDCM) to estimate the directed connections between the left $\mathrm{ACC}$, right ACC, left caudate, right caudate, left DLPFC, left hippocampus, and right hippocampus. We used Bayesian parameter averaging (BPA) to characterize the differences. The BPA results showed hyperconnectivity from the left ACC to right hippocampus and hypoconnectivity from the right ACC to right hippocampus in SZ relatives compared to HCs. The pattern of anterior cingulate cortico-hippocampal connectivity in SZ relatives may be a familial feature of SZ risk, appearing to reflect familial susceptibility for SZ.

Keywords: schizophrenia, first-degree relatives, functional magnetic resonance imaging, effective connectivity, stochastic dynamic causal modeling

\section{INTRODUCTION}

It is well established that familial risk plays a significant role in the etiology of schizophrenia (SZ) through family, adoption, twin, and sibling studies. SZ as a hereditary component affects $0.3 \%$ to $0.7 \%$ of the general population globally according to American Psychiatric Association (APA, 2013), whereas first-degree relatives have a higher risk of developing SZ, with an actual prevalence of approximate 10\% (Lim and Sim, 1992). In genetic epidemiology studies, a 31\% to 58\% concordance rate of SZ exists in monozygotic twins (Tsuang, 2000). It has been demonstrated that genetic 
liability to SZ was $81 \%$ (95\% confidence interval (CI): $73 \%$, $90 \%$ ) based on results from 12 twin studies of SZ (Sullivan et al., 2003). The individual's heritability in liability just partly mediates family history of SZ (Agerbo et al., 2015). Furthermore, brain structural deficits in twins discordant for SZ were more pronounced in monozygotic than in dizygotic twins (Baare et al., 2001; Hulshoff Pol et al., 2004, 2006), suggesting association of cerebral abnormalities with genetic factors for SZ. In our previous studies, we have detected altered brain structure and function in first episode drug-naïve SZ patients (Chang et al., 2015; Cui et al., 2015, 2016; Huang et al., 2015). Thus the question is whether their first-degree relatives present specific alterations of the brain.

During the past 5 years, many structural magnetic resonance imaging (MRI) studies have revealed that gray matter volume, cortical morphological features, and white matter integrity in individuals at high risk of SZ differ from controls, but usually to a lesser extent than in SZ patients, indicating that structural aberrancies may form markers of susceptibility and transition to this disease (Bois et al., 2015b), despite not absolutely consistent findings. For the cerebral morphology, an interrupted cingulate sulcus pattern and paracingulate morphology are associated with increased genetic risk of SZ (Meredith et al., 2012). In the Edinburgh High Risk Study by Lawrie et al. cortical thinning pronounced in the left middle temporal gyrus (Sprooten et al., 2013), as well as longitudinal reductions for volume of the whole brain and bilateral prefrontal and temporal lobes (McIntosh et al., 2011) and cortical surface area prominently in the frontal, cingulate, and occipital lobes (Bois et al., 2015a) were detected in individuals at familial high risk of SZ compared with controls. Also, young relatives of SZ patients showed reduced bilateral hippocampal volume (Thermenos et al., 2013). As reported in a meta-analysis by Cooper et al. (2014), the gray matter volume increased in the left middle frontal gyrus, and decreased in the left thalamus/putamen, insula, and right superior frontal gyrus in high-risk individuals.

With the exception of diverse structural abnormalities, overall, a series of studies have demonstrated functional alterations in relatives of SZ patients at resting state (McIntosh et al., 2006; Hao et al., 2009; Jang et al., 2011; Liao et al., 2012; Su et al., 2013; Zhou et al., 2015) or task state (Whitfield-Gabrieli et al., 2009; Woodward et al., 2009; Rasetti et al., 2011; Stolz et al., 2012), with significant results found in several specific brain regions involving the dorsolateral prefrontal cortex (DLPFC), anterior cingulate cortex (ACC), caudate, and hippocampus.

Notably, the left DLPFC is a featured brain area in SZ relatives. It has been found that familial liability to SZ was associated with decreased gray matter volume of the left DLPFC (McIntosh et al., 2006). Furthermore, healthy siblings of SZ patients showed reduced white matter fractional anisotropy (FA) in the left DLPFC, without significant difference between SZ patients and their siblings (Hao et al., 2009). DLPFC dysfunction has been implicated in the familial susceptibility for SZ (Li and Funahashi, 2015). Aberrant regional function of the left DLPFC was detected by a resting state MRI study on the first-degree relatives of SZ patients
(Liao et al., 2012). When identifying familial vulnerability markers by examining default mode network (DMN) connectivity, posterior cingulate cortex (PCC) seed region connectivity analysis showed reduced functional connectivity in the bilateral DLPFC of relatives (Jang et al., 2011). Unaffected relatives also had impaired connectivity from the left DLPFC to its coordinated regions, distributed in the bilateral caudate, left middle frontal gyrus, and right cerebellum (Su et al., 2013). However, few studies examined connectivity between some of these brain regions in unaffected relatives of SZ patients (Meda et al., 2012; Su et al., 2013), to date, leaving the open question of brain connectivity among these areas in familial high risk individuals.

Although previous studies have identified brain structural and functional abnormalities in frontal and temporal regions, it is still unclear how these regions interacts with each other differently in relatives of SZ patients compared with healthy controls (HCs). In the current study, we used stochastic dynamic causal modeling (sDCM) to investigate directed brain connectivity within a brain network encompasses ACC, caudate, DLPFC and hippocampus. DCM is a technique to investigate brain effective connectivity which refers to the causal influence of one brain region exerts over another or itself (Friston et al., 2003). Compared with functional connectivity analysis which simply measures the correlations between the blood-oxygen-level-dependent (BOLD) signals of different brain regions, effective connectivity analysis is able to further provide us information on how the signals are propagated within a brain network. Understanding the information flow within a brain network is crucial for understanding the neural mechanism of familial susceptibility for SZ. DCM was first invented to model the interactions between brain regions during task performance (Friston et al., 2003). Dauvermann et al. (2013) found decreased thalamocortical connectivity in first- or second-degree relatives of SZ patients using nonlinear deterministic DCM during verbal fluency processing. Recently, traditional deterministic DCM has been extended to stochastic DCM (Daunizeau et al., 2009; Li et al., 2011, 2014) which is also able to model brain effective connectivity at rest ( $\mathrm{Li}$ et al., 2012). Here we used sDCM to identify changes in brain effective connectivity in unaffected first-degree relatives of SZ patients using restingstate fMRI (rsfMRI) data. On the basis of existing evidence that familial risk for SZ appears along with aberrant brain structural and functional alterations involving DLPFC, ACC, caudate, and hippocampus, we hypothesized that effective connectivity among them would also be disrupted in relatives, and provide more accurate parameter estimates ( $\mathrm{Li}$ et al., 2011) compared with conventional deterministic DCM.

\section{MATERIALS AND METHODS}

\section{Subjects}

We assessed $53 \mathrm{HCs}$ and 48 unaffected first-degree relatives of patients with first episode SZ (age- and gender-matched to HCs). The Diagnostic and Statistic Manual of Mental Disorders, 4th 


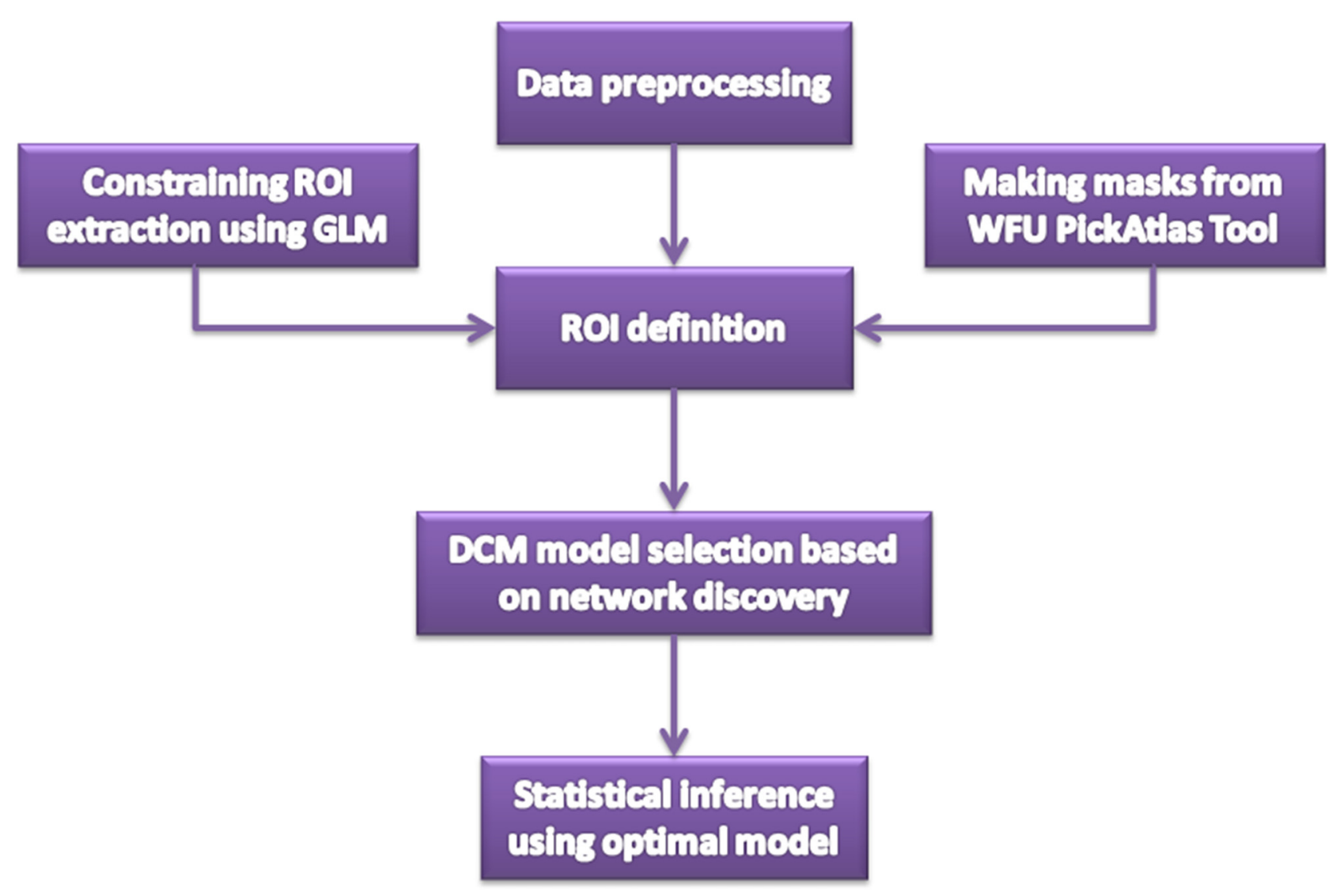

\section{GLM, general linear model; ROI, region of interest}

FIGURE 1 | Steps for data analysis.

edition (DSM-IV), revised criteria (Mittal and Walker, 2011) consensus diagnoses were established by two trained senior clinical psychiatrists with all clinical data and Structured Clinical Interviews for DSM Diagnoses interviews: interrater reliability was higher than $90 \%$ among raters. Relatives of probands were free of Axis 1 psychopathology and not taking psychoactive medications. Participants were recruited via word of mouth and advertisements at the Fourth Military Medical University; all provided written informed consent approved by the institutional review board of Xijing Hospital.

\section{Data Acquisition and Preprocessing}

The resting state fMRI images were collected on the $3.0-\mathrm{T}$ Siemens Magnetom Trio Tim scanner. High-resolution T1-weighted 3D anatomical data were acquired using the 3D magnetization-prepared rapid gradient echo (3D MPRAGE) sequence (repetition time (TR): $2530 \mathrm{~ms}$; echo time (TE): $3.5 \mathrm{~ms}$; flip angle: $7^{\circ}$; field of view (FOV): $256 \times 256 \mathrm{~mm}^{2}$; matrix: $256 \times 256$; slice thickness: $1 \mathrm{~mm}$; section gap:
$0 \mathrm{~mm}$; number of slices: 192). The image resolution was $1 \mathrm{~mm} \times 1 \mathrm{~mm} \times 1 \mathrm{~mm}$. The echo planar imaging (EPI) sequence (TR: $2000 \mathrm{~ms}$; TE: $30 \mathrm{~ms}$; flip angle: 90' FOV: $220 \times 220 \mathrm{~mm}^{2}$; matrix: $64 \times 64$; slice thickness: $4 \mathrm{~mm}$; section gap: $0.6 \mathrm{~mm}$ ) effectively covered the entire brain. Head motion was restricted with a custom-built head-coil foam cushion. During scanning, participants were asked to remain alert with eyes closed and head still. These instructions aided reducing head motion and prevented subjects from falling asleep. All participants were judged as awake and alert at the start and conclusion of the fMRI session. Figure $\mathbf{1}$ is the flowchart for each step. Images were reconstructed offline, and realigned with statistical parametric mapping $\left(\mathrm{SPM} 8^{1}\right)$. The translation/rotation corrections of each participant were examined to exclude excessive head motion $(>2.5 \mathrm{~mm}$ translation and/or $>2.5^{\circ}$ rotation), resulting in that eventual 46 first-degree relatives of SZ patients and $50 \mathrm{HCs}$ were included. A mean functional image volume was constructed for each session from the realigned image volumes to determine parameters for spatial

\footnotetext{
${ }^{1}$ http://www.fil.ion.ucl.ac.uk/spm/software/spm8/
} 


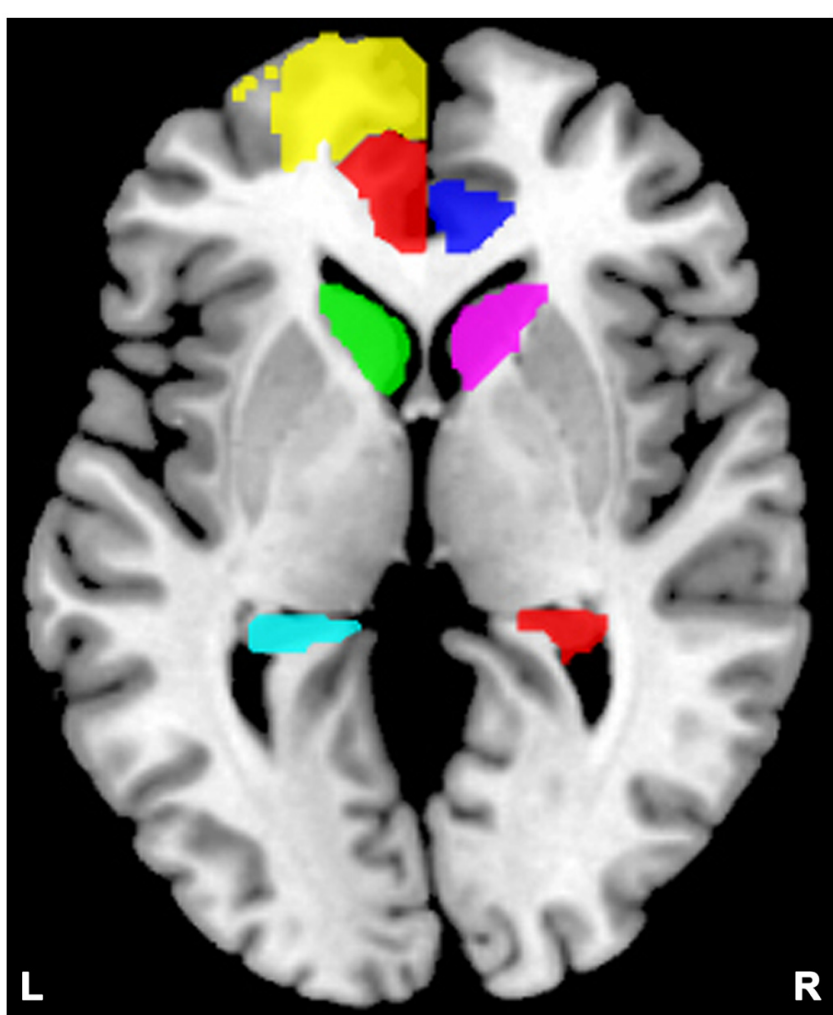

FIGURE 2 | Locations of the masks. Yellow indicates the left dorsolateral prefrontal cortex (DLPFC); semitransparent red indicates the left anterior cingulate cortex (ACC), and blue indicates the right ACC; green indicates the left caudate, and violet indicates the right caudate; cyan indicates the left hippocampus, and red indicates the right hippocampus.

normalization into Montreal Neurological Institute standardized space $^{2}$. Normalization parameters determined for the mean functional volume were applied to the corresponding functional image volumes of each participant, which were smoothed with an $8 \mathrm{~mm}$ full width half maximum (FWHM) Gaussian kernel.

\section{General Linear Model}

In the first-level (within subject) analyses, participant-specific responses were modeled using a general linear model (GLM). The six motion parameters were included to model the movement correlated effects. One constant regressor was used to model the baseline, and cosine basis functions were included in the GLM. The resulting contrast images were then used to constrain the region of interest (ROI) extraction step in the sDCM.

\section{Stochastic Dynamic Causal Modeling}

\section{Regions of Interest}

For each subject, we studied the effective connectivity among seven ROIs including the left DLPFC (consists of Frontal_Sup_L and Frontal_Sup_Medial_L), and the bilateral

\footnotetext{
${ }^{2}$ http://www.mni.mcgill.ca/
}

ACC (Cingulum_Ant_L and Cingulum_Ant_R), caudate nuclei (Caudate_L and Caudate_R), and hippocampi (Hippocampus_L and Hippocampus_R). The left rather than the right DLPFC showed alterations in most studies of SZ relatives during rest condition (McIntosh et al., 2006; Hao et al., 2009; Liao et al., 2012; Su et al., 2013) thereby being chosen as the ROI. For each region, a ROI mask of that region was created by the WFU PickAtlas Tool (Version 3.0.4 ${ }^{3}$ ) and the automated anatomical labeling (AAL) atlas template (Figure 2; Tzourio-Mazoyer et al., 2002; Maldjian et al., 2003, 2004). Subject-specific time series were then extracted based on the ROI mask and the contrast image generated by first-level (within subject) analyses. We then extracted time series from the voxels within the ROI that also showed activation in the contrast image. The first principle component of these time series was finally used to summarize the BOLD response to the ROI.

\section{Model Specification and Parameter Estimation}

In the current study, we aimed to search over all possible models generated from the connections among the seven ROIs. In this case, we did not limit our analysis to simply compare a few competing hypothesis (models). In contrast, we used a data-driven approach to search over all possible models. Specifically, a fully connected model (full model) with bidirectional connections between any pair of regions was constructed for each subject (Figure 3). Parameter estimates and model evidence of the full model was obtained using generalized filtering which is a recently developed scheme for sDCM model inversion and parameter estimation (Friston et al., 2010). After the full model was inverted, we employed a network discovery procedure (Friston et al., 2011) to search for the best reduced model which has the highest model evidence. A reduced model has the same group of ROIs as the full model, but only a subgroup of the connections in the full model (i.e., some of the connections are absent in the reduced model). The network discovery scheme provides approximation of the model evidences of all the possible reduced models without inverting every reduced model. The reduced models and the full model are then scored according to their model evidence. Model which has the highest model evidence was chosen as the winning model. Parameter estimates of the winning model were also obtained using the network discovery scheme and used for group analysis and making inferences on effective connectivity between brain regions.

\section{Group Analysis}

On the basis of sDCM analysis, the strength of connection described the coupling strength according to the rate at which neuronal responses were triggered in the target area (connection strengths are effectively rate constants in $1 / \mathrm{s}, \mathrm{Hz}$; Friston et al., 2003). To see whether these differences could be estimated and detected reliably, we characterized the differences using Bayesian parameter averaging (BPA; Friston et al., 2014; Razi et al., 2015). We used BPA for each group separately after network discovery procedure. We can then go on to discuss the results based on largest two or three connection differences, thereby being as a

\footnotetext{
${ }^{3}$ http://www.nitrc.org/projects/wfu_pickatlas/
} 


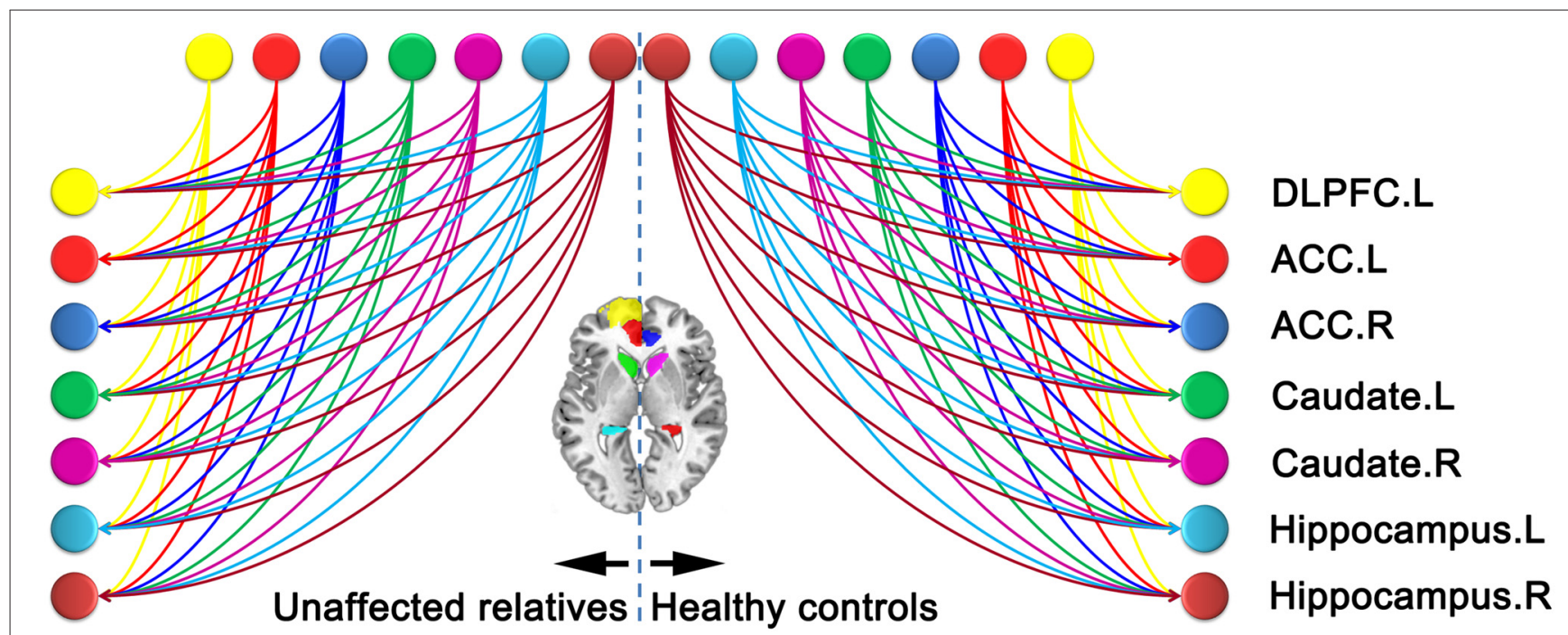

FIGURE 3 | Fully connected model constructed. The lines with arrowheads between distinct region of interests (ROls) refer to the connections in the left panel for relatives of schizophrenia (SZ) patients and right panel for healthy controls (HCs). The color of each node is in line with that of Figure $\mathbf{2}$. ACC, anterior cingulate cortex; DLPFC, dorsolateral prefrontal cortex.

TABLE 1 | Demographical data of the participants.

\begin{tabular}{lcccc}
\hline Variables & $\begin{array}{c}\text { First-degree } \\
\text { relatives of } \\
\text { SZ patients }\end{array}$ & HCs & Statistics & P value \\
& $28 \pm 5$ & $27 \pm 4$ & $t=-0.35$ & 0.73 \\
Age (years) & $22 / 24$ & $31 / 19$ & $\chi^{2}=1.95$ & 0.22 \\
Gender (M/F) & Han (Chinese) & Han (Chinese) & - & - \\
Ethnicity & $46 / 0$ & $50 / 0$ & - & - \\
Handedness (R/L) & $15 \pm 1$ & $15 \pm 2$ & $t=0.23$ & 0.82 \\
Education (years) & $11 / 35$ & $18 / 32$ & $\chi^{2}=1.66$ & 0.27 \\
Smoking status (S/N) & & & &
\end{tabular}

$M$, male; F, female; $R$, right; $L$, left; S, smoker; $N$, nonsmoker.

guiding principle to set the threshold (strength of connections measured in $\mathrm{Hz}$ ).

\section{RESULTS}

\section{Demographical Characteristics}

No significant differences were present between SZ patients' firstdegree relatives and HCs on any demographic variables (Table 1).

\section{Network Discovery-Based Model Selection Results}

The evidence of all reduced models was compared by the network discovery procedure for each group (Figure 4). The left panel is for first-degree relatives of SZ patients and right panel refers to HCs. The procedure selected the fully connected model as the best model with a posterior probability of almost 1 . The fully connected model had 49 parameters describing the extrinsic connections between nodes and the intrinsic (self-connections) within nodes. In Figure 4, the profiles of model evidences are shown with the posterior probability for each model. In both groups, the full model had a log-probability of almost 0 and probability of 1 . Therefore, they shared the identical winning model.

\section{Effectivity Connectivity}

BPA results of the effective connectivity can be seen in Figure 5. When using BPA, in the context of uncovering the group differences, as a guiding principle it would be best to choose top two or three connections and then we set the threshold to $0.06 \mathrm{~Hz}$. SZ patients' relatives exerted increased connection from the left ACC to right hippocampus, but decreased connection from the right ACC to right hippocampus as compared to HCs.

\section{DISCUSSION}

Our study presents sDCM-based effective connectivity outcomes contrasted between first-degree relatives of first episode SZ patients and HCs. As compared with HCs, first-degree relatives who did not show any psychiatric symptoms revealed abnormal connectivity primarily localized to the connections from the bilateral ACC to right hippocampus.

Cognitive deficits are a core characteristic of SZ (Elvevag and Goldberg, 2000), which has been previously observed in biological relatives of SZ patients (Snitz et al., 2006; Bove, 2008; Keshavan et al., 2010; Liao et al., 2012). As well, impaired neural circuitry within the emotion processing has been reported in unaffected siblings of SZ patients (van Buuren et al., 2011; Hanssen et al., 2015). The neural basis of impaired cognition, including emotion processing, in SZ patients and their relatives remains uncertain, thus leaving an open question of whether presence of cognitive and emotional deficits in unaffected firstdegree relatives at high risk for developing SZ suggests genetic basis of SZ symptoms. Determining the neural correlates of 

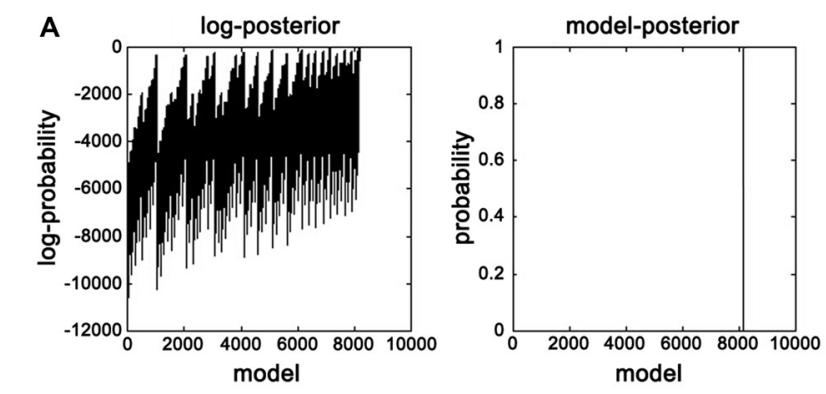

\section{Relatives of SZ patients}
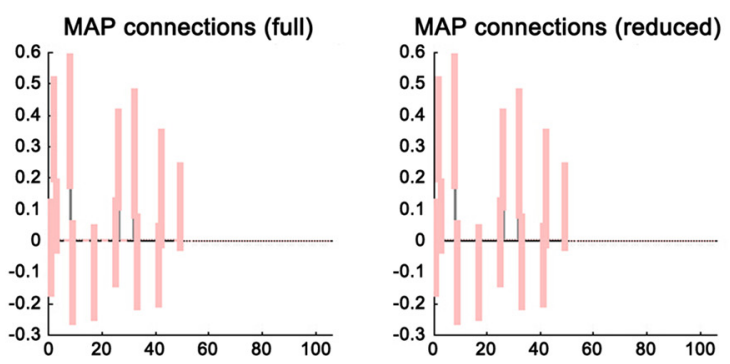

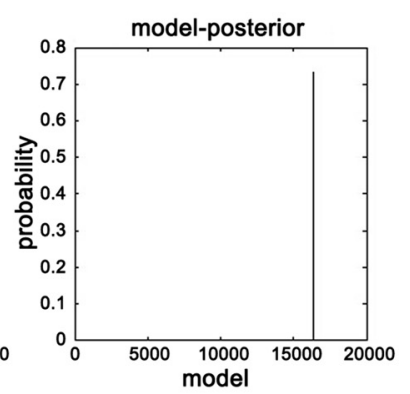

HCs

FIGURE 4 | Results of the post hoc optimization. The corresponding conditional parameter estimates were shown over the 49 (extrinsic and intrinsic) connections in relatives of SZ patients (A) and HCs (B). This figure suggested that the fully connected model was the best explanation for the data.

A

BPA differences

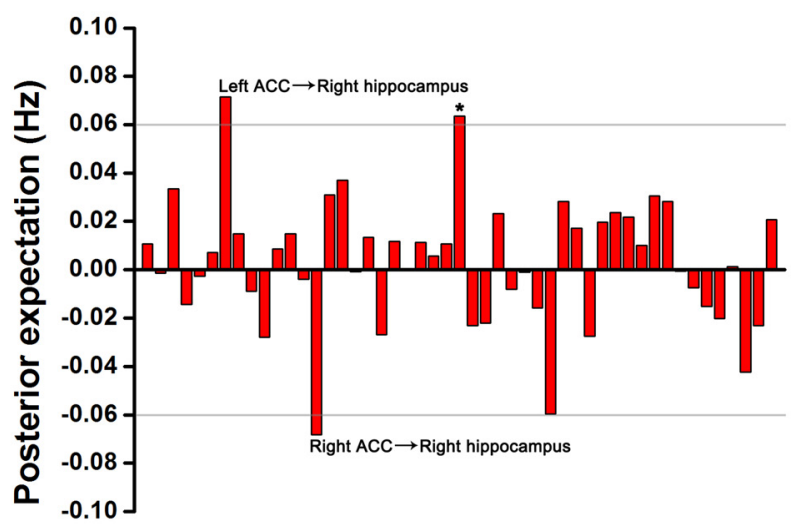

Connections

\section{B}

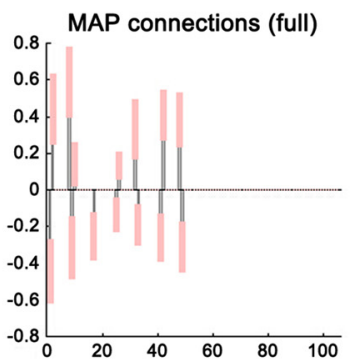

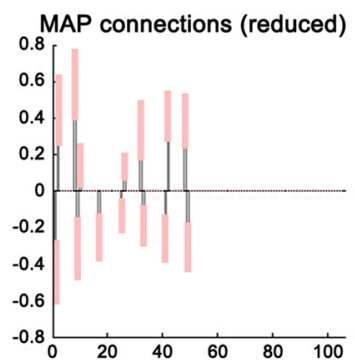

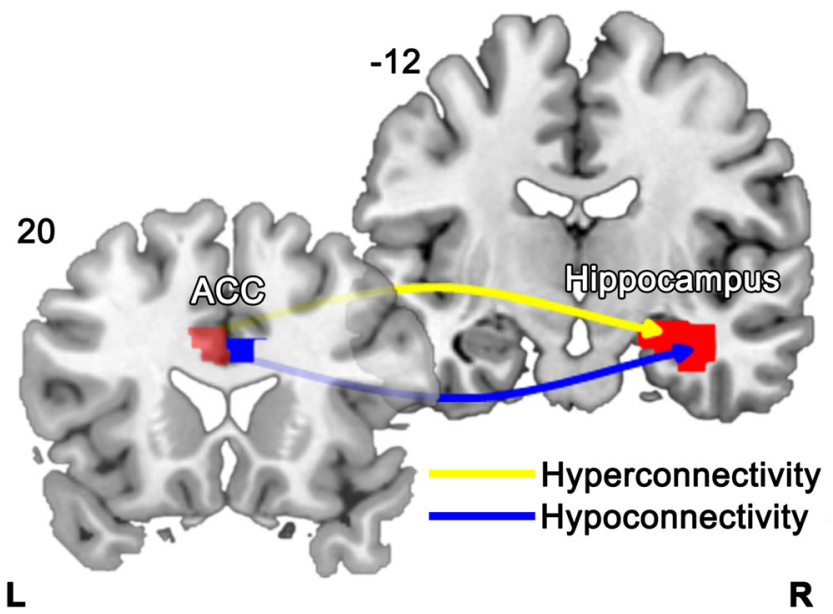

FIGURE 5 | Significant effective connectivity (between the group level) among ROIs in the first-degree relatives of SZ patients and HCs. Bayesian parameter averaging (BPA) of the differences for stochastic dynamic causal modeling (SDCM) shows only those edges on the graph that survive the threshold of $0.06 \mathrm{~Hz}$, i.e., the increased (left ACC-right hippocampus) and decreased (right ACC-right hippocampus) connections in relatives compared to HCs (A). Schematic illustration showing connectivity patterns in first-degree relatives of SZ patients (B). *Indicates self-connection of the left caudate. The slice location (coordinate) is marked in the upper-left.

familial risk for SZ is essential to elucidate the neurobiology for SZ that may aid in the development of novel targeted treatment.

For one thing, relatives of SZ patients exhibited bilateral anterior cingulate cortical dysconnectivity in our present study. Previous studies have demonstrated the important role of the dorsal ACC in cognitive control (Carter and van Veen, 2007) consistently. For these regions, these are association of aberrant activation patterns with deficient 
behavioral performance in SZ (Minzenberg et al., 2009). Most recently, we found altered effective connectivity related to ACC in SZ patients using spectral DCM, indicating anterior cingulate cortico-prefrontal-hippocampal hyperconnectivity (Cui et al., 2015). The effective connectivity and white matter connectivity analysis provides some evidence that weaker connectivity involved in ACC may be the neural basis of specific cognitive impairments in SZ (Wagner et al., 2015). Furthermore, using a regional homogeneity approach, Liao et al. (2012) reported decreased local neural activity in ACC in first-degree relatives of SZ patients along cognitive deficits. When taken with these previous results, our findings in unaffected relatives point to the possibility of altered functional interplay between ACC and hippocampus as the unit responsible for cognition and initial sign for developing SZ.

For another, we detect abnormal connection from ACC to the right hippocampus in relatives of SZ patients. Reduced bilateral hippocampal volume has been observed in young relatives of SZ patients (Keshavan et al., 2002; Thermenos et al., 2013). It has been found smaller hippocampi in relatives of SZ patients (Seidman et al., 2002, 2014; Francis et al., 2013). Furthermore, patients with SZ and their healthy siblings shared disrupted white matter integrity in the hippocampus that may be related to higher risk of healthy siblings to develop SZ (Hao et al., 2009). The hippocampus is part of the hippocampal formation that is comprised of subfields namely the dentate gyrus, subiculum, and pre-subiculum. By means of Van Leemput et al. (2009) method enabling quantification of elusive subfields, reduction in volume of the left and right subicula was observed in familial high risk persons with first-degree relatives suffering from SZ or schizoaffective disorder (Francis et al., 2013). The subibulum could mediate hippocampal-cortical interaction, and is purportedly involved in spatial information processing and memory (O'Mara et al., 2009). In the aforementioned study, verbal memory was impaired and significantly correlated with the subicular volume within the relatives of SZ patients (Francis et al., 2013). Dysconnectivity between DLPFC and hippocampal formation has also been reported in SZ patients (Liu et al., 2014). Accordingly, compromised anterior cingulate corticohippocampal connection links with the risk of developing SZ in individuals at familial high risk.

Moreover, aberrant DLPFC connectivity and familial risk for SZ are closely related in SZ pathophysiology (Hao et al., 2009; Whitfield-Gabrieli et al., 2009; Woodward et al., 2009; Jang et al., 2011; Rasetti et al., 2011; Su et al., 2013). The prefrontal cortex (PFC) is a compartment of the human brain involved in highly diverse processes, ranging from cognition, motivation, emotion, working memory and complex motor activity to social interactions (Ku et al., 2015; Zhou et al., 2015). These aforementioned results in SZ patients and their relatives suggest that neuro-integrative deficits from the DLPFC to other brain regions are likely to be involved in cognitive function and the familial risk for SZ. However, we did not detect significantly different DLPFC-related connectivity in the sample of relatives of SZ patients in our current study. Last but not least, altered caudate nucleus-related connections were not observed in SZ relatives compared to HCs, either. Unaffected relatives from mixed families (with at least one relative with SZ and one with bipolar disorder) showed reductions in bilateral caudate gray matter density (McIntosh et al., 2004). Paradoxically, our results did not show aberrant connections involving caudate nucleus in relatives of $\mathrm{SZ}$ patients. This divergence in findings (i.e., the failure to observe anomalies of connections involved in DLPFC and caudate) could be due to differences in subject selection. In our present study, individuals at high risk for SZ were unaffected first-degree relatives of first episode drug-naïve patients with this illness, rather than mixed first- and seconddegree relatives of treated patients commonly used previously. A possible interpretation is the heritable characteristics of SZ and featured effects of facing patients with diverse symptoms before receiving therapy on these subjects in the current study.

We acknowledge that there were several limitations. First, we enrolled a not so large sample size of subjects in this study. Larger sample is desirable to confirm our present findings. Second, the present study did not involve any behavioral data, i.e., we did not measure the severity of cognitive impairment in the relatives. Currently, we are collecting the behavioral data to clarify the relationship between neuroimaging findings and altered cognition. Third, although a recent study demonstrated both noisy and neural effect of head motion on functional connectivity analysis (Zeng et al., 2014), the current study did not examine the difference of head motion between these two groups. This factor should be taken into account in future research.

Our findings show the pattern of effective connectivity among DLPFC, ACC, hippocampus, and caudate in the familial high risk population of SZ patients, which may be tied to a familial risk of SZ. Specifically, we found that increased effective connectivity from the left ACC to right hippocampus and decreased effective connectivity from the right ACC to right hippocampus in unaffected first-degree relatives of first episode SZ patients. The anterior cingulate cortico-hippocampal dysconnectivity may therefore serve as a potential sign of a general vulnerability to develop SZ.

\section{AUTHOR CONTRIBUTIONS}

HY had full access to all the data in the study and take responsibility for the integrity of the data and the accuracy of the data analysis. Y-BX, CL and L-BC contributed equally to this work. Study concept and design: H-NW, HY. Acquisition, analysis, or interpretation of data: Y-BX, CL, L-BC, JL, FG, LL, T-TL, KL, GC, MX, H-NW, HY. Drafting of the manuscript: Y-BX, CL, L-BC. Critical revision of the manuscript for important intellectual content: Y-BX, CL, L-BC, FG, H-NW, HY. Statistical analysis: CL, L-BC. Administrative, technical, or material support: CL, L-BC, MX. Study supervision: HY.

\section{ACKNOWLEDGMENTS}

This work was supported by the National Key Basic Research and Development Program (973; Grant No. 2011CB707805), National Natural Science Foundation of China (Grant Nos. 
81571651, 81171278 and 81301199), and Fund for the Dissertation Submitted to Fourth Military Medical University for the Academic Degree of Doctor (Grant No. 2014D07). We

\section{REFERENCES}

Agerbo, E., Sullivan, P. F., Vilhjálmsson, B. J., Pedersen, C. B., Mors, O., Borglum, A. D., et al. (2015). Polygenic risk score, parental socioeconomic status, family history of psychiatric disorders and the risk for schizophrenia: a danish population-based study and meta-analysis. JAMA Psychiatry 72, 635-641. doi: 10.1001/jamapsychiatry.2015.0346

APA. (2013). Diagnostic and Statistical Manual of Mental Disorders. 5th Edn. Washington, DC: American Psychiatric Association.

Baare, W. F., van Oel, C. J., Hulshoff Pol, H. E., Schnack, H. G., Durston, S., Sitskoorn, M. M., et al. (2001). Volumes of brain structures in twins discordant for schizophrenia. Arch. Gen. Psychiatry 58, 33-40. doi: 10.1001/archpsyc. 58.1.33

Bois, C., Ronan, L., Levita, L., Whalley, H. C., Giles, S., McIntosh, A. M., et al. (2015a). Cortical surface area differentiates familial high risk individuals who go on to develop schizophrenia. Biol. Psychiatry 78, 413-420. doi: 10.1016/j. biopsych.2014.12.030

Bois, C., Whalley, H. C., McIntosh, A. M., and Lawrie, S. M. (2015b). Structural magnetic resonance imaging markers of susceptibility and transition to schizophrenia: a review of familial and clinical high risk population studies. J. Psychopharmacol. 29, 144-154. doi: 10.1177/0269881114541015

Bove, E. A. (2008). Cognitive performance and basic symptoms in first-degree relatives of schizophrenic patients. Compr. Psychiatry 49, 321-329. doi: 10. 1016/j.comppsych.2008.01.001

Carter, C. S., and van Veen, V. (2007). Anterior cingulate cortex and conflict detection: an update of theory and data. Cogn. Affect. Behav. Neurosci. 7, 367-379. doi: 10.3758/cabn.7.4.367

Chang, X., Xi, Y. B., Cui, L. B., Wang, H. N., Sun, J. B., Zhu, Y. Q., et al. (2015). Distinct inter-hemispheric dysconnectivity in schizophrenia patients with and without auditory verbal hallucinations. Sci. Rep. 5:11218. doi: 10 . 1038/srep11218

Cooper, D., Barker, V., Radua, J., Fusar-Poli, P., and Lawrie, S. M. (2014). Multimodal voxel-based meta-analysis of structural and functional magnetic resonance imaging studies in those at elevated genetic risk of developing schizophrenia. Psychiatry Res. 221, 69-77. doi: 10.1016/j.pscychresns.2013. 07.008

Cui, L. B., Liu, K., Li, C., Wang, L. X., Guo, F., Tian, P., et al. (2016). Putamenrelated regional and network functional deficits in first-episode schizophrenia with auditory verbal hallucinations. Schizophr. Res. 173, 13-22. doi: 10.1016/j. schres.2016.02.039

Cui, L. B., Liu, J., Wang, L. X., Li, C., Xi, Y. B., Guo, F., et al. (2015). Anterior cingulate cortex-related connectivity in first-episode schizophrenia: a spectral dynamic causal modeling study with functional magnetic resonance imaging. Front. Hum. Neurosci. 9:589. doi: 10.3389/fnhum.2015.00589

Daunizeau, J., Friston, K. J., and Kiebel, S. J. (2009). Variational Bayesian identification and prediction of stochastic nonlinear dynamic causal models. Physica D 238, 2089-2118. doi: 10.1016/j.physd.2009.08.002

Dauvermann, M. R., Whalley, H. C., Romaniuk, L., Valton, V., Owens, D. G., Johnstone, E. C., et al. (2013). The application of nonlinear dynamic causal modelling for fMRI in subjects at high genetic risk of schizophrenia. Neuroimage 73, 16-29. doi: 10.1016/j.neuroimage.2013.01.063

Elvevag, B., and Goldberg, T. E. (2000). Cognitive impairment in schizophrenia is the core of the disorder. Crit. Rev. Neurobiol. 14, 1-21. doi: 10. 1615/critrevneurobiol.v14.i1.10

Francis, A. N., Seidman, L. J., Tandon, N., Shenton, M. E., Thermenos, H. W., Mesholam-Gately, R. I., et al. (2013). Reduced subicular subdivisions of the hippocampal formation and verbal declarative memory impairments in young relatives at risk for schizophrenia. Schizophr. Res. 151, 154-157. doi: 10.1016/j. schres.2013.10.002

Friston, K. J., Harrison, L., and Penny, W. (2003). Dynamic causal modelling. Neuroimage 19, 1273-1302. doi: 10.1016/s1053-8119(03)00202-7

Friston, K. J., Kahan, J., Biswal, B., and Razi, A. (2014). A DCM for resting state fMRI. Neuroimage 94, 396-407. doi: 10.1016/j.neuroimage.2013.12.009 are truly grateful to Baojuan $\mathrm{Li}$ from School of Biomedical Engineering, Fourth Military Medical University for her help about the technical guidance and revision.

Friston, K. J., Li, B., Daunizeau, J., and Stephan, K. E. (2011). Network discovery with DCM. Neuroimage 56, 1202-1221. doi: 10.1016/j.neuroimage.2010. 12.039

Friston, K. J., Stephan, K., Li, B. J., and Daunizeau, J. (2010). Generalised filtering. Math. Probl. Eng. 2010:621670. doi: 10.1155/2010/621670

Hanssen, E., van der Velde, J., Gromann, P. M., Shergill, S. S., de Haan, L., Bruggeman, R., et al. (2015). Neural correlates of reward processing in healthy siblings of patients with schizophrenia. Front. Hum. Neurosci. 9:504. doi: 10. 3389/fnhum.2015.00504

Hao, Y., Yan, Q., Liu, H., Xu, L., Xue, Z., Song, X., et al. (2009). Schizophrenia patients and their healthy siblings share disruption of white matter integrity in the left prefrontal cortex and the hippocampus but not the anterior cingulate cortex. Schizophr. Res. 114, 128-135. doi: 10.1016/j.schres.2009.07.001

Huang, P., Xi, Y., Lu, Z. L., Chen, Y., Li, X., Li, W., et al. (2015). Decreased bilateral thalamic gray matter volume in first-episode schizophrenia with prominent hallucinatory symptoms: a volumetric MRI study. Sci. Rep. 5:14505. doi: 10. 1038/srep 14505

Hulshoff Pol, H. E., Brans, R. G., van Haren, N. E., Schnack, H. G., Langen, M., Baare, W. F., et al. (2004). Gray and white matter volume abnormalities in monozygotic and same-gender dizygotic twins discordant for schizophrenia. Biol. Psychiatry 55, 126-130. doi: 10.1016/s0006-3223(03)00728-5

Hulshoff Pol, H. E., Schnack, H. G., Mandl, R. C., Brans, R. G., van Haren, N. E., Baare, W. F., et al. (2006). Gray and white matter density changes in monozygotic and same-sex dizygotic twins discordant for schizophrenia using voxel-based morphometry. Neuroimage 31, 482-488. doi: 10.1016/j. neuroimage.2005.12.056

Jang, J. H., Jung, W. H., Choi, J. S., Choi, C. H., Kang, D. H., Shin, N. Y., et al (2011). Reduced prefrontal functional connectivity in the default mode network is related to greater psychopathology in subjects with high genetic loading for schizophrenia. Schizophr. Res. 127, 58-65. doi: 10.1016/j.schres.2010.12.022

Keshavan, M. S., Dick, E., Mankowski, I., Harenski, K., Montrose, D. M., Diwadkar, V., et al. (2002). Decreased left amygdala and hippocampal volumes in young offspring at risk for schizophrenia. Schizophr. Res. 58, 173-183. doi: 10.1016/s0920-9964(01)00404-2

Keshavan, M. S., Kulkarni, S., Bhojraj, T., Francis, A., Diwadkar, V., Montrose, D. M., et al. (2010). Premorbid cognitive deficits in young relatives of schizophrenia patients. Front. Hum. Neurosci. 3:62. doi: 10.3389/neuro.09. 062.2009

Ku, Y., Bodner, M., and Zhou, Y. D. (2015). Prefrontal cortex and sensory cortices during working memory: quantity and quality. Neurosci. Bull. 31, 175-182. doi: 10.1007/s12264-014-1503-7

Li, B., Daunizeau, J., Stephan, K. E., Penny, W., Hu, D., and Friston, K. (2011). Generalised filtering and stochastic DCM for fMRI. Neuroimage 58, 442-457. doi: 10.1016/j.neuroimage.2011.01.085

Li, B., Friston, K. J., Liu, J., Liu, Y., Zhang, G., Cao, F., et al. (2014). Impaired frontal-basal ganglia connectivity in adolescents with internet addiction. Sci. Rep. 4:5027. doi: 10.1038/srep05027

Li, B. M., and Funahashi, S. (2015). A step forward in the understanding of prefrontal cortical functions. Neurosci. Bull. 31, 161-163. doi: 10.1007/s12264015-1516-2

Li, B., Wang, X., Yao, S., Hu, D., and Friston, K. (2012). Task-dependent modulation of effective connectivity within the default mode network. Front. Psychol. 3:206. doi: 10.3389/fpsyg.2012.00206

Liao, H., Wang, L., Zhou, B., Tang, J., Tan, L., Zhu, X., et al. (2012). A restingstate functional magnetic resonance imaging study on the first-degree relatives of persons with schizophrenia. Brain Imaging Behav. 6, 397-403. doi: 10. 1007/s11682-012-9154-7

Lim, L. C., and Sim, L. P. (1992). The prevalence of schizophrenia in relatives of schizophrenic patients. Singapore Med. J. 33, 645-647.

Liu, B., Zhang, X., Hou, B., Li, J., Qiu, C., Qin, W., et al. (2014). The impact of MIR137 on dorsolateral prefrontal-hippocampal functional connectivity in healthy subjects. Neuropsychopharmacology 39, 2153-2160. doi: 10.1038/npp. 2014.63 
Maldjian, J. A., Laurienti, P. J., and Burdette, J. H. (2004). Precentral gyrus discrepancy in electronic versions of the Talairach atlas. Neuroimage 21, 450-455. doi: 10.1016/j.neuroimage.2003.09.032

Maldjian, J. A., Laurienti, P. J., Kraft, R. A., and Burdette, J. H. (2003). An automated method for neuroanatomic and cytoarchitectonic atlasbased interrogation of fMRI data sets. Neuroimage 19, 1233-1239. doi: 10. 1016/s1053-8119(03)00169-1

McIntosh, A. M., Job, D. E., Moorhead, T. W., Harrison, L. K., Forrester, K., Lawrie, S. M., et al. (2004). Voxel-based morphometry of patients with schizophrenia or bipolar disorder and their unaffected relatives. Biol. Psychiatry 56, 544-552. doi: 10.1016/j.biopsych.2004.07.020

McIntosh, A. M., Job, D. E., Moorhead, W. J., Harrison, L. K., Whalley, H. C., Johnstone, E. C., et al. (2006). Genetic liability to schizophrenia or bipolar disorder and its relationship to brain structure. Am. J. Med. Genet. B Neuropsychiatr. Genet. 141B, 76-83. doi: 10.1002/ajmg.b.30254

McIntosh, A. M., Owens, D. C., Moorhead, W. J., Whalley, H. C., Stanfield, A. C., Hall, J., et al. (2011). Longitudinal volume reductions in people at high genetic risk of schizophrenia as they develop psychosis. Biol. Psychiatry 69, 953-958. doi: 10.1016/j.biopsych.2010.11.003

Meda, S. A., Gill, A., Stevens, M. C., Lorenzoni, R. P., Glahn, D. C., Calhoun, V. D., et al. (2012). Differences in resting-state functional magnetic resonance imaging functional network connectivity between schizophrenia and psychotic bipolar probands and their unaffected first-degree relatives. Biol. Psychiatry 71, 881-889. doi: 10.1016/j.biopsych.2012.01.025

Meredith, S. M., Whyler, N. C., Stanfield, A. C., Chakirova, G., Moorhead, T. W., Job, D. E., et al. (2012). Anterior cingulate morphology in people at genetic high-risk of schizophrenia. Eur. Psychiatry 27, 377-385. doi: 10.1016/j.eurpsy. 2011.11.004

Minzenberg, M. J., Laird, A. R., Thelen, S., Carter, C. S., and Glahn, D. C. (2009). Meta-analysis of 41 functional neuroimaging studies of executive function in schizophrenia. Arch. Gen. Psychiatry 66, 811-822. doi: 10. 1001/archgenpsychiatry.2009.91

Mittal, V. A., and Walker, E. F. (2011). Diagnostic and statistical manual of mental disorders. Psychiatry Res. 189, 158-159. doi: 10.1016/j.psychres.2011.06.006

O’Mara, S. M., Sanchez-Vives, M. V., Brotons-Mas, J. R., and O’Hare, E. (2009). Roles for the subiculum in spatial information processing, memory, motivation and the temporal control of behaviour. Prog. Neuropsychopharmacol. Biol. Psychiatry 33, 782-790. doi: 10.1016/j.pnpbp.2009.03.040

Rasetti, R., Sambataro, F., Chen, Q., Callicott, J. H., Mattay, V. S., and Weinberger, D. R. (2011). Altered cortical network dynamics: a potential intermediate phenotype for schizophrenia and association with ZNF804A. Arch. Gen. Psychiatry 68, 1207-1217. doi: 10.1001/archgenpsychiatry.2011.103

Razi, A., Kahan, J., Rees, G., and Friston, K. J. (2015). Construct validation of a DCM for resting state fMRI. Neuroimage 106, 1-14. doi: 10.1016/j.neuroimage. 2014.11.027

Seidman, L. J., Faraone, S. V., Goldstein, J. M., Kremen, W. S., Horton, N. J., Makris, N., et al. (2002). Left hippocampal volume as a vulnerability indicator for schizophrenia: a magnetic resonance imaging morphometric study of nonpsychotic first-degree relatives. Arch. Gen. Psychiatry 59, 839-849. doi: 10. 1001/archpsyc.59.9.839

Seidman, L. J., Rosso, I. M., Thermenos, H. W., Makris, N., Juelich, R., Gabrieli, J. D., et al. (2014). Medial temporal lobe default mode functioning and hippocampal structure as vulnerability indicators for schizophrenia: a MRI study of non-psychotic adolescent first-degree relatives. Schizophr. Res. 159, 426-434. doi: 10.1016/j.schres.2014.09.011

Snitz, B. E., Macdonald, A. W. 3rd, and Carter, C. S. (2006). Cognitive deficits in unaffected first-degree relatives of schizophrenia patients: a meta-analytic review of putative endophenotypes. Schizophr. Bull. 32, 179-194. doi: 10. 1093/schbul/sbi048

Sprooten, E., Papmeyer, M., Smyth, A. M., Vincenz, D., Honold, S., Conlon, G. A., et al. (2013). Cortical thickness in first-episode schizophrenia patients and individuals at high familial risk: a cross-sectional comparison. Schizophr. Res. 151, 259-264. doi: 10.1016/j.schres.2013.09.024
Stolz, E., Pancholi, K. M., Goradia, D. D., Paul, S., Keshavan, M. S., Nimgaonkar, V. L., et al. (2012). Brain activation patterns during visual episodic memory processing among first-degree relatives of schizophrenia subjects. Neuroimage 63, 1154-1161. doi: 10.1016/j.neuroimage.2012.08.030

Su, T. W., Lan, T. H., Hsu, T. W., Biswal, B. B., Tsai, P. J., Lin, W. C., et al. (2013). Reduced neuro-integration from the dorsolateral prefrontal cortex to the whole brain and executive dysfunction in schizophrenia patients and their relatives. Schizophr. Res. 148, 50-58. doi: 10.1016/j.schres.2013. 05.005

Sullivan, P. F., Kendler, K. S., and Neale, M. C. (2003). Schizophrenia as a complex trait: evidence from a meta-analysis of twin studies. Arch. Gen. Psychiatry 60, 1187-1192. doi: 10.1001/archpsyc.60.12.1187

Thermenos, H. W., Keshavan, M. S., Juelich, R. J., Molokotos, E., WhitfieldGabrieli, S., Brent, B. K., et al. (2013). A review of neuroimaging studies of young relatives of individuals with schizophrenia: a developmental perspective from schizotaxia to schizophrenia. Am. J. Med. Genet. B Neuropsychiatr. Genet. 162B, 604-635. doi: 10.1002/ajmg.b.32170

Tsuang, M. (2000). Schizophrenia: genes and environment. Biol. Psychiatry 47, 210-220. doi: 10.1016/s0006-3223(99)00289-9

Tzourio-Mazoyer, N., Landeau, B., Papathanassiou, D., Crivello, F., Etard, O., Delcroix, N., et al. (2002). Automated anatomical labeling of activations in SPM using a macroscopic anatomical parcellation of the MNI MRI single-subject brain. Neuroimage 15, 273-289. doi: 10.1006/nimg.2001.0978

van Buuren, M., Vink, M., Rapcencu, A. E., and Kahn, R. S. (2011). Exaggerated brain activation during emotion processing in unaffected siblings of patients with schizophrenia. Biol. Psychiatry 70, 81-87. doi: 10.1016/j.biopsych.2011. 03.011

Van Leemput, K., Bakkour, A., Benner, T., Wiggins, G., Wald, L. L., Augustinack, J., et al. (2009). Automated segmentation of hippocampal subfields from ultra-high resolution in vivo MRI. Hippocampus 19, 549-557. doi: 10.1002/hipo.20615

Wagner, G., De la Cruz, F., Schachtzabel, C., Gullmar, D., Schultz, C. C., Schlosser, R. G., et al. (2015). Structural and functional dysconnectivity of the fronto-thalamic system in schizophrenia: a DCM-DTI study. Cortex 66, 35-45. doi: 10.1016/j.cortex.2015.02.004

Whitfield-Gabrieli, S., Thermenos, H. W., Milanovic, S., Tsuang, M. T., Faraone, S. V., McCarley, R. W., et al. (2009). Hyperactivity and hyperconnectivity of the default network in schizophrenia and in firstdegree relatives of persons with schizophrenia. Proc. Natl. Acad. Sci. U S A 106 , 1279-1284. doi: 10.1073/pnas.0809141106

Woodward, N. D., Waldie, B., Rogers, B., Tibbo, P., Seres, P., and Purdon, S. E. (2009). Abnormal prefrontal cortical activity and connectivity during response selection in first episode psychosis, chronic schizophrenia and unaffected siblings of individuals with schizophrenia. Schizophr. Res. 109, 182-190. doi: 10. 1016/j.schres.2008.11.028

Zeng, L. L., Wang, D., Fox, M. D., Sabuncu, M., Hu, D., Ge, M., et al. (2014). Neurobiological basis of head motion in brain imaging. Proc. Natl. Acad. Sci. US A 111, 6058-6062. doi: 10.1073/pnas.1317424111

Zhou, Y., Fan, L., Qiu, C., and Jiang, T. (2015). Prefrontal cortex and the dysconnectivity hypothesis of schizophrenia. Neurosci. Bull. 31, 207-219. doi: $10.1007 /$ s12264-014-1502-8

Conflict of Interest Statement: The authors declare that the research was conducted in the absence of any commercial or financial relationships that could be construed as a potential conflict of interest.

Copyright (c) $2016 \mathrm{Xi}, \mathrm{Li}$, Cui, Liu, Guo, Li, Liu, Liu, Chen, Xi, Wang and Yin. This is an open-access article distributed under the terms of the Creative Commons Attribution License (CC BY). The use, distribution and reproduction in other forums is permitted, provided the original author(s) or licensor are credited and that the original publication in this journal is cited, in accordance with accepted academic practice. No use, distribution or reproduction is permitted which does not comply with these terms. 